\title{
Learning relationships from theory to design
}

\author{
C. J. H. Fowler*I and J.T. Mayes** \\ *Advanced Communications Research, BT Adastral Park \\ **Learning and Educational Development, Glasgow Caledonian University, \\ email: chris.fuwler@btcom
}

This paper attempts to bridge the psychological and anthropological views of situated learning by focusing on the concept of a learning relationship, and by exploiting this concept in our framework for the design of learning technology. We employ Wenger's (1998) concept of communities of practice to give emphasis to social identification as a central aspect of learning, which should crucially influence our thinking about the design of learning environments. We describe learning relationships in terms of form (oneto-one, one-to-many etc.), nature (explorative, formative and comparative), distance (first-, second-order), and context, and we describe a first attempt at an empirical approach to their identification and measurement.

\section{Introduction}

Over the last five years we have seen a very significant increase in the use of Information Communication Technologies (ICT) in schools, colleges and university. For example in 1998, there were over 195 accredited US universities offering a thousand or more distance learning courses (Philips and Yager, 1998). By no means were all of these new courses associated with educational innovation. The speed and ease of implementation of Webbased approaches, in particular, is resulting in design by imitation of current courses and methods, with a real lack of innovation or utilization of the power inherent in technologybased learning. Although matters are improving (see for example Brown, 1999), part of the reason for this failure to innovate is, we argue, because of the large gap between theory and practice.

This paper sets out to bridge what might be regarded as the psychological and anthropological views of situated learning by focusing on the concept of learning relationships. The aim is to consider how, in designing learning environments and tasks, we 
might usefully shift our focus away from the design of activities and examine more carefully what it is that motivates learners to engage in the learning task in the first place. What is it that would encourage them to strive for mastery? We ask what it is that the individual learner characteristically brings to the activity and to the environment, beyond the rather unproven variables of learning style. Finally we consider, with an example from a current project, how the concept of learning relationships might be elaborated empirically and used in the design of learning technology.

The theoretical underpinning of attempts to design learning environments has recently witnessed two distinct shifts of emphasis. First, there has been a shift from a representational view of learning in which an acquisition metaphor guided design (e.g. Anderson, Boyle, Farrell and Reiser, 1990), to a constructivist view in which learning is primarily developed through activity (Papert, 1990). Brown, Collins and Duguid (1989) argued that we should consider concepts as tools, to be understood through use, rather than as self-contained entities to be delivered through instruction. A second shift, however, has been away from a focus on the individual, towards a new emphasis on social contexts for learning (Glaser, 1990). There are at least two aspects to this. First, we have seen for about fifteen years a new emphasis on learning through collaboration and co-operation (see Kaye, 1992), but, even more importantly perhaps, came the notion of situated learning (Lave, 1988; Lave and Wenger, 1991; Suchman 1987).

As Barab and Duffy (1999) point out, there are at least two 'flavours' to situated learning. One, following Resnick's (1987) articulation of the nature of informal, out-of-school learning, and Cole and Scribner's (1974) approach to the cognitive implications of formal and informal learning, can be regarded as a socio-psychological view of situativity. They emphasized the importance of context-dependent learning in informal settings. Children in traditional societies learn by observing others, a form of apprenticeship where the knowledge often remains tacit. In contrast, formal education removes the child from context by locating learning in schools. The separation of where you learn from what you learn can be seen as playing an important part in developing abstract thinking in the child, but a growing dissatisfaction with formal schooling has led to a 'participation' metaphor replacing the 'acquisition' metaphor (Sfard, 1998). See Laurillard (1993) for a discussion of the 'abstraction' issue in the context of university education.

This activity-based view of situated learning led to the design of what Barab and Duffy (following Senge, 1994) call 'practice fields'. These represent constructivist tasks in which every effort is made to make the learning activity authentic to the social context in which the skills or knowledge are normally embedded. Examples of approaches to the design of practice fields are problem-based learning (Savery and Duffy, 1996), anchored instruction (CTGV, 1993) and cognitive apprenticeship (Collins et al., 1989). Here, the main design emphasis is on the relationship between the nature of the learning task in educational or training environments, and its characteristics when situated in real use. This connection between the classroom and the real world through 'practice fields' has certain cognitive implications. It is unclear, for example, how children abstract out general principles and avoid becoming 'context-bound' (cf. Cole and Scribner, 1974).

The second view of situativity, however, is a social anthropological one in which the influence of a wider social context is emphasized (Lave and Wenger, 1991). Here we find 
the concept of a community of practice introduced. With it comes an emphasis on the practitioner's relationship with a wider but identifiable group of people rather than the relationship of the activity itself to the wider practice, even though it is the practice itself that identifies the community. This provides a different perspective on what is 'situated'. For Lave (1997) and Wenger (1998) it is not just the meaning to be attached to an activity that is derived from a community of practice, but a much more fundamental aspect. The individual's identity is shaped by the relationship. Lave and Wenger's perspective, however, emphasizes the stable and long-term nature of communities of practice. In our view this restricts the potential usefulness of the idea for the design of learning environments, where short-term and more fragile groups may nevertheless exert a powerful influence on the motivation to learn. Indeed, the social psychological literature on social identification (Turner, 1991; Hogg and Adams, 1988) seems to demonstrate that temporary but real social identities can be created through group membership.

The starting point for this paper is a description of two conceptual frameworks. Wenger (1998) describes how communities of practice underpin learning in both formal and informal contexts. Mayes and Fowler (1999) map a cognitive-constructivist account of learning onto the design of learning environments.

\section{Wenger's conceptualization}

For Wenger, knowledge is a matter of competence in a valued enterprise. The value is given by social participation - in particular, by being an active participant in the practices of social communities, and by constructing an identity in relation to each community. Participating in a community - it may be a project team, say, or a member of a professional group - is both a kind of action and a form of belonging. Wenger's social theory of learning, therefore, recognizes both strands of situativity described above: meaning is given both to the situated activities themselves and to the process of social identification which drives the learners' activity.

Wenger argues that the social production of meaning is the relevant unit of analysis for practice. Meaning is continually negotiated through the processes of participation and reification. It is worth quoting Wenger directly on participation:

Participation is an active process, but I will reserve the term for actors who are members of social communities. For instance, I will not say that a computer 'participates' in a community of practice, even though it may be part of that process and play an active role in getting things done ... In this regard what I take to characterise participation is the possibility of mutual recognition ... What we recognise has to do with our mutual ability to negotiate meaning ... The relations between parents and children, or between workers and supervisor, are mutual in the sense that participants shape each others' experiences of meaning.

For Wenger, participation is not the same thing as collaboration.' It goes beyond direct engagement in specific activities, and is 'not something we turn on and off'. Wenger argues that our engagement with tasks is social, even where it does not directly involve any kind of explicit dialogue. 
Being in a hotel room by yourself preparing a set of slides for a presentation the next morning may not seem like a particularly social event, yet its meaning is fundamentally social. Not only is the audience with you as you attempt to make your points understandable, but your colleagues are there too, looking over your shoulder, as it were, representing for you your sense of accountability to the professional standards of your community.

We can participate in global communities of practice, but more generally we tend to engage with local ones. How can we identify that a community of practice has formed? Wenger suggests that we must look for sustained mutual relationships, which will tend to involve shared ways of engaging in doing things together, and the rapid flow of information. The discourse in such a community will have the appearance of an ongoing process, with many shared assumptions and an absence of introductory preambles. There will be jargon, inside jokes, many communication shortcuts, and the rapid setup of a problem to be discussed.

For Wenger, there are three stages of coming to belong to a community of practice:

- imagination: through orientation and exploration, we identify with a community of practice;

- engagement: through participating in a community we value, we come to belong to that community;

- alignment: we connect to a new framework of convergence.

Wenger articulates the same basic point that this paper is arguing, namely that issues of education should be addressed first and foremost in terms of identities and modes of belonging, and only secondarily in terms of skills and information. This view encourages us to consider educational designs not just in terms of techniques for supporting the construction of knowledge (let alone in terms of delivery of a curriculum), but more generally in terms of their effects on the formation of identities. What does this mean for design? Wenger goes some way towards operationalizing his framework (although not very far) by proposing that students need:

- places of engagement;

- materials and experiences with which to build an identity;

- ways of making their actions matter.

The constructivist approach requires the design of tasks which are personally meaningful for learners. Wenger's conceptualization of communities of practice gives us a way of defining personal meaning in a way that is not just circular. However, it is not a description of learning per se, or of how people learn together. It provides a very high-level design heuristic and in that sense it tells us where we should start looking for design principles particularly within an organizational context.

\section{A framework for the design of learning technology}

When BT became interested in designing and building online educational services, it soon became apparent that it was hard to move from theoretical concepts, e.g. constructivism or conceptualization, to a set of design principles or guidelines that could help engineer the next generation of educational technology. We needed conceptual frameworks that bridged 
the theory and design. Mayes (1995) offered such a framework. This framework described three broad modes of learning and then mapped these onto appropriate design principles. The modes or stages of learning were:

- Conceptualization: the coming into contact with other people's concepts.

- Construction: the building and testing of one's knowledge through the performance of meaningful tasks.

- Dialogue: the debate and discussion that results in the creation of new concepts.

It is important to note that 'conceptualization' is about other people's concepts, 'construction' is about building knowledge from combining your own and other people's concepts into something meaningful. 'Dialogue' refers back to the creation of new concepts (rather than knowledge) that then triggers another cycle of the reconceptualization process.

Fowler and Mayes (1997) have extended the notion of dialogue to include dialogues or learning conversations for clarification and confirmation at the conceptualization stage, and dialogue for co-operation and collaboration at the construction stage. The framework proposes that each learning stage should be associated with a different kind of pedagogy, which in turn implies a different kind of learning environment and a different form of courseware. At conceptualization, the learner tends to rely on traditional forms of courseware which explain and illuminate the concepts in the subject matter, typically building an exposition through textbooks, multimedia or simulations. This type of courseware was referred to as primary. Secondary courseware took the form of materials or tools that supported 'learning by doing', while tertiary courseware referred to the captured dialogues which gave rise to significant learning episodes and the availability of this material to others in the support of vicarious learning (McKendree, Stenning, Mayes, Lee, and Cox, 1998).

The framework can be developed, by elaborating the role of dialogue. We have previously emphasized the importance of dialogue in learning, elevating it above, or at least level with, conceptualization as a pedagogical design principle. Dialogue provides the vehicle for conceptual movement. It facilitates the transition between the stages and the advance from one reconceptualization cycle to the next. ${ }^{2}$ To emphasize the central role of dialogue even further we now view it not as a separate stage, but as integral to the whole cycle. The third stage we have now labelled 'identification'. At the identification stage, the learner has reached a sufficient level of understanding to be able to relate to other conceptualizations and thus begin the process again. By calling this process identification it is intended to emphasize our belief that it is social in origin, and can only be understood in terms of the learner's relationships with others. It makes the important design point that this stage cannot be fully understood by knowing all about the interactions between the subject matter, the tasks, or the pedagogical environment. The nature of the interactions between individual learners and other people now comes fully into focus.

\section{Learning relationships: a new conceptualization}

Wenger's account paints a rich picture of the social process of belonging and provides an explanation of personal motivation through identification with the practices of communities. People are not motivated to learn per se, but are motivated to join a community 
of practice - an end that can be best achieved through learning. It is this richer social context and explanation of motivation that are lacking in our previous framework.

The two frameworks impact on design in different ways. For Wenger the main implication of his analysis is for organizations. For the present authors, the design goals are pedagogical. The combination of pedagogical and organizational design principles offers the potential of innovative and potentially powerful applications.

Wenger's account of the stages of coming to belong to a community of practice parallels the stages of learning in our own account. His imagination stage, where the individual begins to identify the boundaries of a community of practice, involves a process of conceptualization. Equally engagement and construction are both about 'doing and discovering', and alignment and identification are stages which adjust understanding to a wider context.

A learning relationship exists when we learn from, or through, others. Such relationships will vary according to the characteristics of the groups involved, the context within which they operate, and the strength of the relationships. These relationships may be one of three different forms: one-to-one (e.g. parent to child); one-to-many (e.g. teacher to learners); and many-to-many (e.g. learning in peer groups or networks). The strength and effectiveness of the learning will also vary within the different kinds of relationships. These types of relationship vary according to the nature of the learning experience and are referred to as explorative, formative and comparative learning relationships, and they are described in more detail below.

An explorative learning relationship is about discovery. In our previous account it involves the discovery of other peoples' concepts, and for Wenger, the discovery of the practice boundaries. This relationship is often very descriptive (discovering the 'what' more than the 'why'), although some level of explanation will be involved, and asymmetrical in that most of the information flow is one way (from the 'outside in'). A formative learning relationship focuses more on the building of understanding through guided activity, and this is achieved through a constructive approach - the building and testing of hypotheses about the world, and about the nature of practice. This relationship is likely to be balanced or symmetrical, with outputs from the learner matched by feedback on performance. The third kind of learning relationship, comparative, characterizes a relationship that occurs once a level of expertize has been achieved or when an individual becomes accepted as more than a peripheral member of a community of practice. It is a relationship that allows the learner to identify their state of knowledge with others, or to align their practice with that of a community or organization. The primary purpose of a comparative relationship is not necessarily to acquire new knowledge, but to position and adjust existing knowledge by comparison with other knowledge states. The asymmetry is therefore in the opposite direction to the explorative stage (i.e. 'inside out'), and is less about acquisition and more about tuning and maintenance of the knowledge or practice. Such relationships may take on a defensive nature that can account for both the emotional charge of some of the dialogue that takes place and the resistance to change of some communities of practice.

Social network theory provides us with a method for describing and modelling learning relationships within given boundaries. The nodes in such a network represent the learners, and the links between them indicate that a relationship exists. Further, the concept of 
learning relationships recognizes that such relationships in themselves exist within a wider network. A relationship can be defined in terms of the distance between the two nodes. Learning relationships (regardless of type) are all first-order relationships. Clearly, secondorder and other more distant relationships will influence these relationships and their requirements. For example, the relationship between a student and a teacher is, in terms of learning, a first-order relationship. However, that relationship will be influenced by the teacher's relationship with the year co-ordinator (second-order), and the year coordinator's relationship with the headteacher (third-order) and so on. It is assumed that the effective boundary of a learning relationship does not exceed a third-order relationship, although a community of practice could extend beyond that (see Figure 1). The community formed by a bounded learning relationship can be regarded as a microcommunity of learners, within a community of practice. As well as determining the boundaries of the micro-community, another reason for measuring the different orders of relationship is that it may help to identify ways of supporting a first-order relationship. The basic rationale for a social network analysis is to focus attention on the relationships that exist, and to identify those that do not.

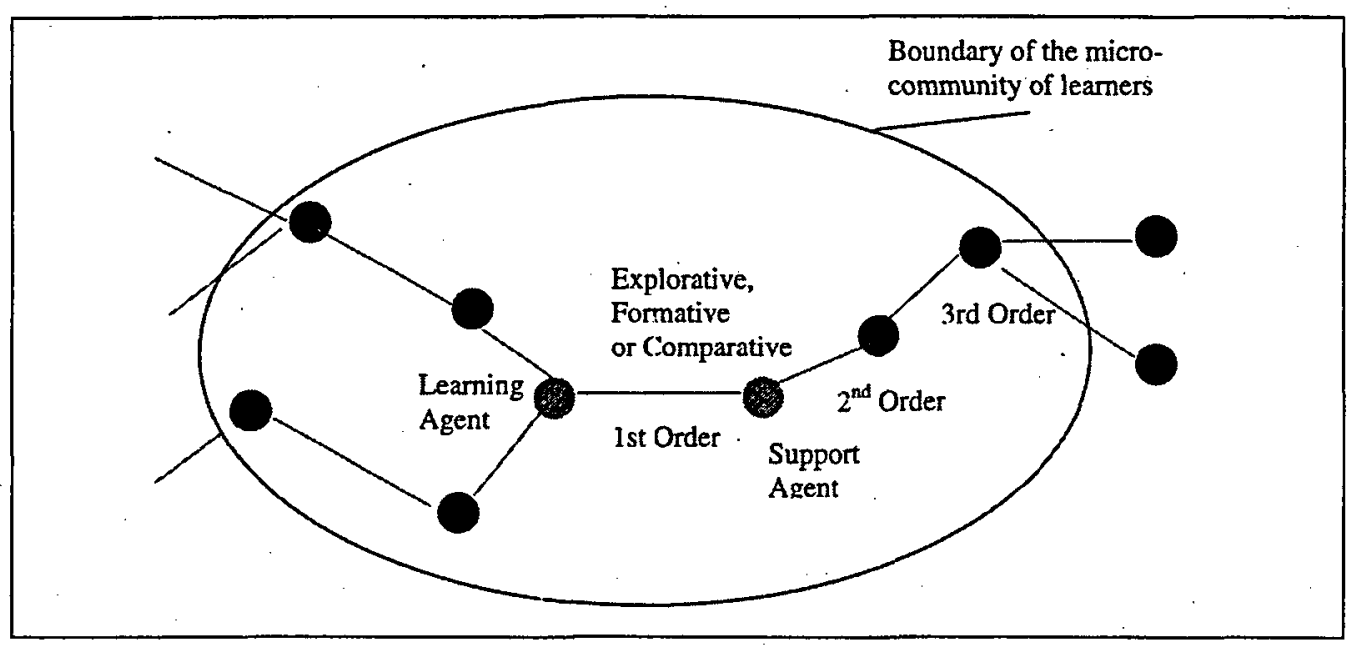

Figure 1. A typical representation of a leaming relationship

These characteristics of the learning relationship are summarized below:

- Forms: one-to-one; one-to-many; many-to-many;

- Types: explorative, formative, comparative;

- Distance: first-order; second-order; third-order;

- Context: social groups; socio-political climate; wider community of practice.

The nodes within a learning relationship or the wider learning network are referred to as agents. The learner in the relationship is called a 'learning agent', and the facilitator is called a 'support agent'. It is important to distinguish between the different functions 
provided by the learning agent. Any agent must be a knowledge resource, able to sustain a dialogue with the learner, and able to guide and support the learning tasks. These are the characteristics which have previously been used to specify the requirements for an intelligent tutoring system (Wenger, 1987). Any agent must be able potentially to satisfy these criteria, although in any particular learning relationship these conditions may not be met. A crucial issue to address is the extent to which the characteristics of an agent in a learning relationship can be provided through technology. This is a fundamental question about the role of learning technology, under which most of the design issues for computerbased learning environments can be subsumed. What aspects of effective learning support absolutely require a human relationship?

\section{What next?}

Our next steps are to operationalize the concept and to collect empirical evidence to test, refine or refute it. We intend to explore the learning relationships of sixteen-year-olds attending schools in three different European countries (UK, Finland and Portugal). By using countries from the north, middle and south of Europe we are also hoping to identify any key cultural variables that may influence the nature and form of the learning relationships.

Learning relationships will be identified using a questionnaire, and represented visually in a learning network using a proprietary drawing tool. The drawing tool also supports annotations of the relationships with both specific data (e.g. descriptions of the learners) out to more general data (e.g. descriptions of the schools and cultures). Once the main support agent of the learner has been identified, then the relationship between the learner and support agent (e.g. teacher, parent or friend) can be classified. The two agents undertake a task and their learning behaviours are classified as one of the three learning types througn the use of a checklist. The relationship type is then noted on the learning relationship diagram. Data are also being collected about the individual learner, their families, school and wider communities.

Once all the data have been collected these will be analysed using a multi-level modelling technique (Goldstein, 1995). This technique allows one to assess the different factors affecting the relationships. For example, strong learning relationships may only be found for learners from certain types of families or who attend schools with a particular learning ethos.

If learning relationships prove to be a powerful factor in learning, then the concept will be used to rethink how we define online educational services. Future services that are sensitive to learning relationships may look for ways to optimize configurations so that key agents are connected and their relationship is maximized to enhance learning. It is planned to build and test such a prototype service for home-school communities in the UK, Finland and Portugal.

The emphasis of this empirical work is to explore whether or not learning relationships can provide us with a sufficiently robust analytical model for determining requirements for, and evaluation of, learning networks. Where such networks exist, the concept can help us identify relationships that should be formed, optimized or even terminated. An IP-based service based on learning relationships may be just what is required to move us away from 
the overemphasis on content or the 'teacher-in-the-box' services that are currently prevalent in the market.

\section{Conclusions}

By subjecting the framework developed in our earlier work (Mayes and Fowler, 1999) to the perspective offered by Wenger's work on communities of learning we have shifted the focus of our work in the following ways:

- moving the emphasis of learning away from the 'what' we learn to the 'who' we learn from;

- more directly addressing the key factor of motivation to learn;

- providing a bridging framework from which both pedagogical and organization design can be derived;

- offering insights into how best to manage change within our learning organizations.

This new emphasis helps to reinforce the point that learning within organizations and within traditional educational settings requires a common explanatory framework.

It remains to be seen whether we can move from the conceptual framework described in this paper to the specification of a design methodology which properly embeds the technological support of learning in the wider social context in which the identities of learners are shaped.

\section{Acknowledgements}

This project is partially funded by the European Institute for Research and Strategic Studies in Telecommunication (EURESCOM) and involves partners from BT (UK), Portugal Telecoms, Telenor (Norway); Finnet Group (Finland), Slovakia Telecoms and Icelandic Telecoms. The authors are also grateful to the many colleagues who have contributed through discussion. In particular, we like to thank the members of the Vicarious Learning Project based in Glasgow Caledonian and Edinburgh Universities and BT's Education and Training Research team at Adastral Park. We are also grateful to Christina Knussen for helpful advice on Social Identification literature.

\section{Notes}

1 Chris Fowler also works at the Institute of Education, University of London, in a half-time capacity.

2 The original framework was based on a simple goal-action feedback loop called the conceptualization cycle. As with Miller, Galanter and Pribram's TOTE units (1960) it was seen as a building block from which more and more complex descriptions of learning could be progressively constructed. (Larger TOTE units could be built from smaller, a process that modelled the way in which practice builds larger skill sequences.) The framework referred to here is the second-order description, where the action stage was unpacked into stages of conceptualization, construction and dialogue. The framework is intended less as a description of learning and more as an illustration of design principles that can be distilled from a range of psychological, social and educational literatures. 


\section{References}

Anderson, J. R., Boyle, C. F., Farrell, R. and Reiser, B. J. (1987), 'Cognitive principles in the design of computer tutors', in P. Morris (ed.), Modeling Cognition, New York: Wiley.

Barab, S. and Duffy, T. (in press), 'From practice fields to communities of practice', in D. Jonassen and S. Land (eds.), Theoretical Foundations of Learning Environments, Mahwah, NJ: Erlbaum.

Brown, J. S., Collins, A. and Duguid, P. (1989), 'Situated cognition and the culture of learning', Educational Researcher, 18, 32-42.

Brown, S. (1999), 'Virtual university: real challenges', in B. Collis and R. Oliver (eds.), Proceedings of ED-MEDIA 1999: World Conference on Educational Multimedia and Telecommunications, AACE.

Cognition and Technology Group at Vanderbilt (1990), 'Anchored instruction and its relation to situated learning', Educational Researcher, 19, 2-10.

Cole, M. and Scribner, S. (1974), Culture and Thought: A Psychological Introduction, New York: Wiley.

Collins, A., Brown, J. S. and Newman, S. E. (1989), 'Cognitive apprenticeship: teaching the crafts of reading, writing and mathematics', in L. B. Resnick (ed.), Knowing, Learning and Instruction: Essays in Honour of Robert Glaser, Hillsdale, NJ: Erlbaum.

Fowler, C. J. H. and Mayes, J. T. (1997), 'Applying telepresence to education', in BT Technology Journal, 14, 188-95.

Garton, L., Haythornthwaite, C., and Wellman, B. (1997), 'Studying on-line social networks', Journal of Computer Mediated Communication, 3 (1).

Glaser, R. (1990), 'The re-emergence of learning theory within instructional research', American Psychologist, 45, 1, 29-39.

Goldstein, H. (1995), Multilevel Statistical Models, London: Edward Arnold.

Hog, M. A. and Adams, D. (1988), Social Identification, London: Routledge.

Kaye, A.R. (ed.) (1992), Collaborative Learning through Computer Conferencing: The Najaden Papers, Heidelberg, FRG: Springer Verlag.

Laumann, E., Marsden, P. and Prensky, D. (1983), 'The boundary specification problem in network analysis', in R. Burt and M. Minor (eds.), Applied Network Analysis, Beverly Hills, CA: Sage.

Laurillard, D. (1993), Rethinking University Teaching: A Framework for the Effective Use of Educational Technology, London: Routledge.

Lave, J. and Wenger, E. (1991), Situated Learning: Legitimate Peripheral Participation, Cambridge: Cambridge University Press.

Lave, J. (1988), Cognition in Practice, Cambridge: Cambridge University Press.

Lave, J. (1997), 'The culture of acquisition and the practice of understanding', in 
D. Kirshner and J. A. Whitson (eds.), Situated Cognition: Social, Semiotic and Psychological Perspectives, Mahwah, NJ: Erlbaum.

Mayes, J. T. and Fowler, C. J. H. (1999), 'Learning technology and usability: a framework for understanding courseware', Interacting with Computers, 11, 485-97.

Mayes, J. T. (1995), 'Learning technologies and Groundhog Day', in W. Strang, V. B. Simpson and D. Slater (eds.), Hypermedia at Work: Practice and Theory in Higher Education, Canterbury: University of Kent Press.

McKendree, J., Stenning, K., Mayes, J. T., Lee, J. and Cox, R. (1998), 'Why observing a dialogue may benefit learning', Journal of Computer Assisted Learning, 14, 110-19.

Miller, G., Galanter, E. and Pribram, K. (1960), Plans and the Structure of Behavior, New York: Holt, Reinhart \& Winston.

Papert, S. (1990), 'An introduction to the fifth anniversary collection', in I. Harel (ed.), Constructionist Learning, MIT Media Laboratory, Cambridge: MA.

Philips, V. and Yager, C. (1998), Best Distance Learning Graduate Schools: Earning your Degree without Leaving Home, Princeton Review: Random House.

Resnick, L. B. (1987), 'Learning in school and out', Educational Researcher, 16, 13-20.

Savery, J., and Duffy, T. (1996), 'Problem-based learning: an instructional model and its constructivist framework', in B. Wilson (ed.), Constructivist Learning Environments: Case Studies in Instructional Design, Englewood Cliffs, NJ: Educational Technology Publications.

Senge, P. (1994), The Fifth Discipline Fieldbook: Strategies and Tools for Building a Learning Organisation, New York: Doubleday.

Sfard, A. (1998), 'On two metaphors for learning and the dangers of choosing just one', Educational Researcher, 27, 4-13.

Suchman, L. (1987), Plans and Situated Actions: The Problem of Human-Machine Interaction, Cambridge: Cambridge University Press.

Turner, J. C. (1991), Social Influence, Milton Keynes: Open University Press.

Wellman, B. and Berkowitz, S. D. (1988), Social Structures: A Network Approach, Cambridge: Cambridge University Press.

Wenger, E. (1987), Artificial Intelligence and Tutoring Systems: Computational and Cognitive Approaches to the Communication of Knowledge, Los Altos, CA: Morgan Kaufmann.

Wenger, E. (1998), Communities of Practice: Learning, Meaning and Identity, Cambridge: Cambridge University Press. 